\title{
Milton Friedman: \\ EL ECONOMISTA ANTES DEL VIAJE DEFINITIVO
}

\author{
POR Julio G. Andújar Scheker ${ }^{\mathrm{a}}$
}

Recibido: mayo de 2019 • Aceptado: junio de 2019

Cómo citar: Andújar Scheker, J. G. (2019). Milton Friedman: el economista antes del viaje definitivo. Ciencia, Economía y Negocios, 3(2), 9-41.

Doi: https://doi.org/10.22206/ceyn.2019.v3i2.pp9-41

\section{Resumen}

Milton Friedman es conocido por muchos, para bien o para mal, como el más alto exponente de la Escuela de Chicago, némesis de la dominante escuela keynesiana durante los años de postguerra. Controversial, polémico y siempre presto a defender sus ideas, Friedman fue más que eso. Fue una estrella con luz propia que escribió más de 200 artículos y que revolucionó la teoría económica en sus fases monetaria, real, metodológica y filosófica (Beyer, 1995). Influyó de forma decisiva en el debate de políticas públicas. Su impronta quedó marcada en economías tan distantes como la china y la chilena y en ramas de esta ciencia tan apartadas como la monetaria y la economía de la educación. Todo esto y mucho más se sabe de uno de los economistas más influyentes del siglo XX. De su vida previa al viaje definitivo a Chicago se conoce menos. Este documento explora esa historia en un tour de force desde sus orígenes y su estructura familiar hasta la actividad frenética de los años de la Segunda Guerra Mundial y la postguerra, cubriendo sus años de formación, las ideas que lo influenciaron, sus mentores, sus comienzos en el mercado laboral y su visión académica fundamentada en una extraña mezcla de Chicago con Columbia, entre otros.

Palabras clave: Milton Friedman; Historia; Economía Monetaria; Monetarismo.

Códigos: JEL: B30, B31, B32.

${ }^{a}$ Andújar, Julio G.: Universidad Iberoamericana (UNIBE), Empírica: Centro de Aplicaciones Económicas y Banco Central de la República Dominicana (BCRD). Dirección: Apartado Postal 1347, Av. Pedro H. Ureña esq. Leopoldo Navarro, Santo Domingo, República Dominicana. Tel.: +1 809 221-9111, (e-mail: j.andujar@bancentral.gov.do). Lo expresado en este artículo es exclusiva responsabilidad del autor. 


\title{
Milton Friedman: The economist BEFORE THE DEFINITIVE JOURNEY
}

\author{
By Julio G. ANDÚJAR SCHEKER
}

Received: may, 2019 • Approved: jun, 2019

\begin{abstract}
Milton Friedman is known by many, for better or worse, as the highest exponent of the Chicago School, the nemesis of the dominant Keynesian school during the Post-War years. Controversial and always ready to defend his ideas, Friedman was more than that. He was a star with its light who wrote more than 200 articles and revolutionized economic theory in its monetary, real, methodological and philosophical phases (Beyer, 1995). He decisively influenced the public policy debate. His imprint was marked in economies as distant as China and Chile and in branches of economic science as far apart as monetary and educational economics. All this and much more is known as one of the most influential economists of the twentieth century. However, from his life before the definitive trip to Chicago, little is known. This document explores that story in a tour de force from its origins and family structure to the frantic activity of the World War II and Post War years, covering its formative years, the ideas that influenced him, his mentors, his beginnings in the labor market and his academic vision based on a strange mixture of Chicago with Columbia, among others.
\end{abstract}

Keywords: Milton Friedman; History; Monetary Economics; Monetarism.

JEL codes: B30, B31, B32. 


\section{Introducción}

Si tomamos como punto de partida la publicación en la Escocia del siglo XVIII de la obra cumbre de Adam Smith, La riqueza de las naciones, la economía es una ciencia joven. Cierto es que las ideas planteadas por Smith y el resto de los clásicos durante este período pueden rastrearse tan lejos como a los albores de la antigua civilización griega. Es así como, en su enciclopédica obra sobre la historia del pensamiento económico, Schumpeter (1954) identifica rastros de las ideas económicas modernas en los trabajos de Aristóteles y Platón. No obstante, estos orígenes remotos, difícilmente puede hablarse de una ciencia económica como un todo organizado antes de la obra cumbre del filósofo escocés.

Las ideas de Smith y los clásicos, con sus múltiples similitudes y diferencias, dominaron la teoría económica por más de 150 años. Desde el optimismo inicial de Smith, bajo el supuesto de que la especialización en el trabajo llevaba a rendimientos crecientesy mejoras en productividad, hasta el incorregible pesimismo de pensadores como Malthus, Ricardo y Marx, el sistema económico era visto como la suma de sus partes; un ente que operaba en complejo equilibrio y que era capaz de autocorregirse ante cualquier desvío inesperado de este "estado natural de cosas" (Smith, [1776] 2004; Ricardo, [1817] 2004). Aunque existía la posibilidad de que esos desvíos terminaran irremediablemente en algún tipo de colapso (Malthus, [1798] 2015; Marx, [1867] 2011), no había la opción de intervenir para evitar o suavizar los vaivenes del ciclo económico y solo quedaba sentarse a esperar la autocorrección o el colapso irremediable.

Fue la Gran Depresión de 1929 el evento que probó que la economía podía languidecer por siempre en una especie de limbo si no se le ofrecía cura al cuerpo enfermo. La cura para esa enfermedad fue descubierta por el más reputado macroeconomista de todos los tiempos: John Maynard Keynes. Con la aparición de la Teoría general del empleo, el dinero y el interés (Keynes, 1936), los economistas descubrieron la economía del desequilibrio y se alinearon en masa tras este poderoso torrente de ideas. Salvo unas pocas excepciones, 
la profesión en general adoptó alguna forma de keynesianismo. Entre los pocos herejes, inmunes a este cuerpo de ideas, llegaría a sobresalir un joven que apenas se recibía de maestro en Economía en la Universidad de Chicago en esos tiempos. Ese joven, Milton Friedman, se convertiría con el tiempo en el más alto exponente de la Escuela de Chicago, némesis de la dominante escuela keynesiana.

Krugman (2007) afirma que, si Keynes fue el Martín Lutero de la economía, protestando contra la capilla Clásica, Friedman se convirtió con el tiempo en su Ignacio de Loyola, el gran reformador que dio nueva vida a ideas que se creían enterradas. Sin dudas, Friedman fue más que eso. Fue una estrella con luz propia que escribió más de 200 artículos y que revolucionó la teoría económica en sus fases monetaria, real, metodológica y filosófica (Beyer, 1995). Fue además de un extraordinario polemista, un economista de los que abandonaba periódicamente el confinamiento académico para hacer públicas sus ideas e influir de forma decisiva en el debate de políticas. Su impronta ha quedado marcada en ramas de la economía tan apartadas como la monetaria y la economía de la educación. Sus sugerencias de políticas públicas tuvieron acogida en economías tan distantes como la china y la chilena.

Hoy, a más de cien años de su nacimiento, es propicio explorar su pensamiento, su filosofía y su trayectoria, pero desde una óptica un tanto distinta a la tradicional. Se trata de hacer un viaje a los primeros años, a las influencias del contorno familiar y a los años de formación. Una exploración del entorno que lo formó y de la coyuntura que le tocó vivir antes de emprender el viaje definitivo a Chicago. En forma sucinta, en eso consiste este documento.

La segunda sección se dedica a los años de formación familiar y escolar, abarcando el período transcurrido desde su nacimiento en Brooklyn hasta su graduación de bachiller en el poblado de Rahway, en el estado de Nueva Jersey. La sección tres comprende sus años de estudios universitarios en la Universidad de Rutgers, a medio camino entre las matemáticas, la economía y las ciencias actuariales. La cuarta sección se enfoca en los años de estudios graduados en Chicago y Columbia y en cómo ese coctel formativo definió su metodología 
económica. En la quinta parte se explora su vida laboral luego de finalizada la maestría, un período rico en experiencias académicas y de políticas públicas que, sin embargo, le obligó a posponer la obtención de su doctorado por más de una década. En la sexta sección se analiza el retorno a Chicago, el viaje definitivo que le llevaría junto a otros colegas a transformar la facultad de economía, dándole forma a la escuela de pensamiento que conocemos hoy. Por último, la séptima sección concluye el documento.

\section{De Brooklyn a Rahway: los años de formación familiar}

Poco antes de que terminara el siglo XIx, la ciudad de Nueva York, compuesta principalmente por Manhattan y una gran parte de lo que hoy es el Bronx, se transformó en una mega urbe al incorporar a su centro administrativo la ciudad de Brooklyn y los poblados rurales de Queens y Staten Island. Al momento de esa fusión, Brooklyn era una de las cinco ciudades más grandes de Estados Unidos, un centro industrial importante con grandes empresas como la farmacéutica Pfizer y un puerto de relevancia con una infraestructura envidiable para el transporte marítimo.

En esta pujante ciudad se conocieron en esa época Jean Saul Friedman y Sarah Ethel Landau, inmigrantes judíos recién llegados de Carpatio-Rumania, una provincia del Imperio Astro-Húngaro situada en lo que actualmente es Ucrania. Jean Saul y Sarah Ethel se unieron en matrimonio y vivieron en Brooklyn el tiempo suficiente para tener cuatro hijos. El más pequeño de todos y el único varón, Milton Friedman, nació el 31 de julio de 1912.

No es difícil imaginar lo complejo que sería para una pareja joven de migrantes judíos, provenientes del otro lado del mundo y con seguras precariedades económicas, levantar cuatro hijos en una ciudad como Brooklyn, con puentes colgantes enormes, con un sistema moderno de trenes subterráneos, con grandes museos e impresionantes estadios deportivos. Por esta razón, no sorprende que apenas un año después 
del nacimiento del pequeño Milton la familia decidiera buscar un espacio más acogedor para asentarse.

Es así como los Friedman llegan a un pequeño poblado industrial, a menos de 20 millas de la ciudad de Nueva York. Aunque pequeño de tamaño, Rahway era pujante en esos tiempos. Tenía un comercio amplio con la metrópolis a través del río Rahway o de las líneas de ferrocarriles que comunicaban a Nueva York con Filadelfia. Fabricaba carruajes, libros, artículos relacionados con la música y productos de consumo masivo. Además, había acogido recientemente a la multinacional farmacéutica Merck, de origen alemán.

De acuerdo al propio Friedman, los primeros años en Rahway fueron difíciles. Su padre tuvo numerosos empleos sin un éxito aparente y su madre trató de abrirse paso en el negocio de las ventas al detalle ${ }^{1}$. Quizás el negocio familiar de mayor duración fue una pequeña heladería en el centro del pueblo. A pesar de las limitaciones económicas, la posibilidad de educarse para el pequeño Milton nunca estuvo en dudas. Junto a sus hermanas, asistió a la escuela pública de Rahway, distinguiéndose como estudiante meritorio desde un principio.

En 1928, cuando Friedman cursaba el último año del bachillerato, su padre Jean Saul murió de angina, dejando a la familia en una situación de incertidumbre económica. Friedman aún no cumplía 16 años y era claro que su madre haría lo que estuviese a su alcance para evitar una deserción escolar y para insertar a su hijo de alguna forma en el sistema universitario. Lógicamente, del deseo a la realidad hay un trecho. Más que los esfuerzos familiares para financiar los estudios del joven Friedman, fueron sus propias habilidades matemáticas las que le abrieron las puertas a una carrera universitaria. Gracias a sus destrezas cuantitativas Friedman se ganó una beca del estado de New Jersey para estudiar en la Universidad de Rutgers.

\footnotetext{
${ }^{1}$ Ver el discurso autobiográfico de Friedman al recibir el Premio Nobel de Economía en 1976.
} 


\section{Rutgers: donde todo comenzó}

Al margen de las razones obvias de la cercanía a casa y de la ayuda económica que proporcionaba la beca, uno se pregunta por qué razón Friedman, hábil con las matemáticas y campeón de debate en la secundaria de Rahway, escogió a Rutgers para sus estudios universitarios. Más aún, es comprensible que se tengan dudas sobre qué lo hizo torcer camino y abandonar una potencial carrera de actuario, luego de tomar los exámenes correspondientes, por una incierta mezcla de matemáticas y economía, disciplinas que no resultaban particularmente atractivas para quienes contrataban en el mercado de trabajo en ese entonces.

Parecería sensato atribuir estas decisiones, que serían claves en el futuro profesional de Friedman, a dos razones. Por un lado, Rutgers tenía una historia importante de éxitos matemáticos y era un entorno ideal para un estudiante con inquietudes numéricas. Por otro lado, entre los miembros de su facultad de profesores, Rutgers tenía dos economistas que ejercerían una marcada influencia en el joven Friedman: un estudiante de doctorado de la Universidad de Columbia, Arthur Burns, quien con el paso del tiempo llegaría a ser la cabeza de la Reserva Federal de los Estados Unidos; y un estudiante graduado de la Universidad de Chicago, Homer Jones, visto por Friedman "como un joven profundo, celoso de su libertad individual y opuesto a la planificación social (Friedman, 1977).

El prestigio matemático de Rutgers durante esos años queda de manifiesto en Weibel (1995), un breve ensayo histórico sobre la fortaleza matemática de la casa de altos estudios a la que acudió Friedman, poco antes de la Gran Depresión. Según Weibel, el primer profesor contratado en la historia de la universidad en 1809, incluso antes de que esta asumiera el nombre de Rutgers, fue el matemático Robert Adrain. Casi ochenta años después, George William Hill (18381914) desarrolló en Rutgers la teoría de ecuaciones diferenciales lineales. En este ambiente, donde la ascendencia de la matemática era un hecho incuestionable, Friedman recibió en 1932 el Bradley Math Prize, un premio a la excelencia matemática que desde 1926 la 
Universidad de Rutgers viene otorgando de manera ininterrumpida a su estudiante de grado más distinguido en esta disciplina.

La matemática de Rutgers sentó los cimientos para que años después, como economista, Friedman incorporara ese arsenal a su forma de hacer teoría. No obstante, queda la pregunta sobre las fuerzas que influyeron para que al final de su estadía en Nueva Jersey Friedman optara por la economía, descartando para siempre el sueño familiar de hacerlo un actuario.

Las evidencias sobre la influencia de Arthur Burns y Homer Jones en esta decisión son abundantes. Friedman fue marcado por las distintas personalidades de sus mentores y probablemente por esto se formó a medio camino entre Chicago, la casa de Jones y Columbia, el hogar de Arthur Burns. No obstante, atribuirle exclusivamente a la influencia de sus mentores la decisión de Friedman de abandonar la carrera de actuario para eventualmente optar por la carrera de economía, una disciplina relativamente joven y con un mercado laboral más estrecho, sería un exceso.

Lo cierto es que en sus años de Rutgers (1929-1932), Friedman vivió de cerca los problemas y las vicisitudes generados por la Gran Depresión. Es factible pensar que, en adición al deseo de sus mentores de que escogiera de forma definitiva el camino de la economía, Friedman tomó su decisión por una inescapable necesidad de comprender lo que estaba ocurriendo en su mundo. Sin duda, fue seducido por la necesidad de entender cómo se generaban las grandes crisis económicas y de dónde provenía su inmenso poder para hacer daño. Es por esa razón que, una vez graduado, dedicó años a estudiar las causas y los efectos de la Gran Depresión.

\section{Los primeros años de estudios graduados (1932-1935)}

\section{IV.1. El Chicago que lo recibió}

Es una realidad innegable que antes de marcharse a Chicago, en 1932, la vida del joven Friedman había transcurrido en un mundo 
relativamente pequeño. De Brooklyn a Rahway y de Rahway a Rutgers no había más que unos pocos cientos de kilómetros. Ese era el mundo de Milton Friedman. Viajar a Chicago sería una experiencia como ninguna otra experimentada en su corta vida. La influencia de Homer Jones en esta decisión transcendental queda fuera de toda discusión. Friedman, impresionado por algunos elementos de la personalidad de su profesor de Seguros y Estadísticas en Rutgers, optó por moverse por primera vez al oeste del Río Delaware.

Friedman (1976) describe algunos rasgos de la personalidad y de la forma de ver el mundo de su mentor, los cuales fueron determinantes en la elección de Chicago. Además de la solidez teórica de Jones, el joven economista resalta su mente crítica, su deseo permanente de aprender y su capacidad de aterrizar complejos conceptos económicos. Otro elemento que resultó atractivo para Friedman fue la visión del mundo que tenía su mentor. Como todo buen discípulo de Frank H. Knight, economista fundador de la vieja escuela de Chicago, Jones consideraba el capitalismo la mejor forma para organizar una economía.

Es claro que, en una época marcada por la Gran Depresión, las ideas de Knight y Jones, aunque decrecientes en popularidad, interesaron al joven Friedman. Esto no quiere decir que la visión del mundo de Chicago fuese un elemento clave en su mudanza al oeste del Río Delaware. En esa etapa de su vida Friedman estaba muy lejos de convertirse en un acólito de la libertad individual y el mercado. De hecho, en una entrevista con el conocido economista John Taylor (febrero, 2001), Friedman afirma que su visión de la economía en ese entonces "era más técnica que filosófica" y que estaba más cerca de una visión socialista del mundo que de cualquier otra ideología.

Bajo esta premisa, es posible que además de la influencia de Homer Jones, el otro elemento determinante en la decisión de Friedman de unirse a Chicago fuese el ofrecimiento de una beca completa de estudios. Durante sus años en Rutgers, Friedman tomó varios empleos de verano, trabajando como dependiente de tiendas en el sector comercial. Su situación económica no había cambiado mucho desde la muerte de su padre y su origen semita cerraba más puertas de 
las que abría. Así, la beca que le consiguió Jones debió ser un alivio a aquella situación precaria, a la vez que ofrecía la oportunidad de estudiar una disciplina interesante en un lugar bien reputado.

Su primera estadía en Chicago duró poco menos de dos años. Fue un tiempo donde Friedman repartió su carga académica proporcionalmente entre cursos de economía, por un lado, y cursos de matemática y estadística, por el otro. Del lado matemático y estadístico, la mayor influencia provino de Henry Schultz, quien al finalizar sus estudios le conseguiría la beca para marcharse a Columbia. Del lado económico, la figura determinante en su formación fue Jacob Viner, la otra gran personalidad de Chicago a principios de la década de 1930.

La influencia de Viner en esos años fue, sin duda, muy superior a la ejercida por Schultz. De hecho, Columbia tuvo que ver más en la formación estadística de Friedman que Chicago, como se verá más adelante. En total, Friedman tomó tres cursos graduados con Viner durante su primera instancia en la Universidad de Chicago, siendo el más relevante en su formación Teoría de Precios, primera clase que debía enfrentar cualquier estudiante graduado en Chicago en la década de 1930 (Hammond, 2001).

Samuelson (1972) describe este curso introductorio de teoría económica de Viner como una experiencia única. Demandante, comprehensivo y un reto para cualquier aspirante a tomarlo (no todos podían entrar), el curso de Viner marcó a una generación de grandes economistas. Menos dogmático y más ecléctico que Knight, Viner fue determinante para que Friedman adoptara una forma muy propia de abordar temas de teoría económica ${ }^{2}$. El desarrollo posterior de sus teorías del consumo, del ingreso permanente y de algunos de sus aportes en teoría monetaria indican que Friedman aprovechó muy bien las enseñanzas de Viner, así como su posición siempre cuestionadora y provocativa en el análisis económico.

\footnotetext{
${ }^{2}$ Además de la formación teórica, el curso de Viner dejó algo más importante a Friedman. Allí conoció a Rose Director, la hermana de un joven profesor de la universidad, Aaron Director, quien unos años después se convertiría en su esposa y en coautora de varias publicaciones (Beyer, 1995).
} 
Sin embargo, dos años fueron suficientes para que Friedman decidiera complementar su experiencia teórica de Chicago con algo más práctico. Es así como en 1933 decide marcharse a Columbia, el hogar de Arthur Burns, donde agregaría a su maletín de herramientas el conocimiento empírico y la visión institucional del mundo. En ese momento, Henry Schultz partía a Europa aprovechando un año sabático, no sin antes gestionar una beca de Columbia para que su estudiante favorito pudiera completar su formación estadística y matemática con uno de los grandes nombres de la época: Harold Hotelling. El propósito de Schultz era que a su vuelta del viejo continente se encontraran de nuevo en Chicago, esta vez con el ojo en el doctorado.

\section{IV.2. De Chicago a Columbia: en busca del empirismo perdido}

Una vez en Columbia, el interés intelectual del joven Friedman se movió de la teoría económica a las estadísticas y a la economía matemática. Allí fue recibido por Harold Hotelling, un famoso estadístico y matemático egresado de Princeton que publicó casi noventa artículos y que definió con sus trabajos seminales al menos seis áreas de estudios que al día de hoy representan la agenda de trabajo de numerosos economistas y estadísticos (Arrow, 1974). Friedman afirma que estudiar en Chicago y en Columbia fue para él una combinación ideal (Friedman \& Friedman, 1999). Chicago le permitió hacerse de una sólida formación en teoría económica. Columbia, por otro lado, le proveyó la parte empírica, dotándole además de los elementos básicos del institucionalismo americano (Hammond, 2001).

En Columbia, de nueve cursos posibles, Friedman tomó cuatro de ellos con Hotelling. Esta experiencia le sirvió tanto para sus primeros trabajos, muy centrados en el área de las estadísticas, como para sus primeros aportes científicos en la metodología y práctica de la economía. En adición a los cursos tomados con Hotelling, el joven Friedman se inscribió en las clases sobre el ciclo económico que dictaba el institucionalista Wesley Clark Mitchell, fundador 
del famoso Bureau Nacional de Investigación Económica (NBER), organismo que define, aun al día de hoy, el estado del ciclo en que se encuentra la economía de Estados Unidos en un momento dado.

Además de Wesley C. Mitchell, otro institucionalista, John M. Clark, tuvo al joven Friedman como su estudiante. De esta forma, Friedman experimentó el institucionalismo americano directamente de dos de los tres fundadores del movimiento intelectual ${ }^{3}$. Quizás la interpretación más apropiada de la visión económica de Friedman en aquel entonces era una mezcla de teoría económica proveniente de Chicago, un conocimiento empírico amplio provisto por Harold Hotelling en Columbia y una perspectiva institucional adquirida bajo la tutela de Mitchel y Clark. A lo largo de su carrera como economista, estos tres elementos se verían reflejados en sus investigaciones una y otra vez.

\section{IV.3. De Columbia a Chicago: cumpliendo una promesa}

Cumpliendo la promesa que le hiciera a su mentor Henry Shultz, Friedman dejó Columbia y retornó a Chicago en 1934. Durante este período que duraría algo más de un año, Friedman tomó pocos cursos y estuvo más bien concentrado en su trabajo de tesis de maestría. Schultz, su mentor y jefe en el Laboratorio de Estadísticas de Chicago, era también su principal consejero de tesis, un estudio empírico sobre la relación entre los precios de las acciones y las ganancias de la industria del ferrocarril entre 1921 y 1931.

Fuera de estas experiencias, quizás el elemento más importante de su retorno a Chicago fue el inicio de una amistad que perduraría toda la vida con dos economistas, Allen Wallis y George Stigler. Los vínculos de Friedman con Wallis y Stigler irían más allá de relaciones labores estrechas en al menos cinco instituciones. Se expandirían hasta el relanzamiento de la escuela de Chicago, como la cuna del liberalismo en el mundo académico.

Al igual que Friedman, Wallis había sido uno de los talentos captados

\footnotetext{
${ }^{3}$ El otro autor es Walton Hamilton, creador del manifiesto institucionalista de 1919: The Institutional Approach to Economic Theory.
} 
para realizar estudios graduados en economía en la Universidad de Chicago a partir de 1932. Llama la atención que el Departamento de Economía de Chicago se empeñó en reclutarlos a pesar de provenir de áreas de estudios y de instituciones marcadamente diferentes. Friedman, como se ha visto, llegó de Rutgers, donde sus pretensiones de ser actuario habían sido vencidas por los cursos de matemáticas y economía que se ofrecían en esta universidad de la costa este de Estados Unidos. Wallis, por su lado, fue reclutado en la Universidad de Minnesota, en el medio oeste norteamericano, donde había obtenido un título en psicología con concentración en sociología.

Aparentemente, durante esos años la Universidad de Chicago estaba más interesada en la capacidad potencial de sus postulantes, pura y simple, y no en su historia personal, sus intereses o en la formación que habían recibido previo a su reclutamiento. Un año antes de la entrada de Friedman como estudiante graduado en la Universidad de Chicago, había sido enganchado el tercer mosquetero, Stigler, quien provenía de la Universidad de Northwestern donde había obtenido una Maestría en Administración de Empresas (MBA), luego de recibir su licenciatura en la Universidad de Washington, en el estado de donde era oriundo. Friedman, Wallis y Stigler, una tríada fundamental en el posicionamiento de Chicago como prototipo de la academia liberal, no podían ser más diferentes. Chicago logró juntar al matemático, al psicólogo-sociólogo y al potencial hombre de negocios.

Es probable que entre los tres nuevos amigos Friedman fuera quien se encontrara más lejos de las ideas centrales de la filosofía de Chicago. Ya sabemos que al momento de partir de Rutgers, en 1932, Friedman se describía más que nada como un "socialista". Durante su primer año en Chicago, Friedman tomó tantos cursos de matemática aplicada como los que seleccionó de teoría económica (Hammond, 2001). Su interés en el análisis cuantitativo continuó en Columbia al año siguiente, dedicándose de forma casi exclusiva a estudiar estadísticas con Harold Hotelling. Dada su mayor exposición durante este período a cursos de matemáticas y estadísticas que a la economía de Chicago, es probable que, en 1934, Friedman aún mantuviera una 
gran parte de sus simpatías "socialistas".

Una experiencia muy diferente fue la de George Stigler. Se puede decir que, desde su arribo a Chicago, Stigler se identificó con la filosofía de su nueva escuela. Para comenzar, seleccionó como asesor de su tesis doctoral a la figura más emblemática del mundo liberal, Frank H. Knight. Considerado el principal padrino de la escuela de Chicago, Knight guio la tesis de Stigler sobre las teorías neoclásicas de producción y distribución. En sus intercambios, transmitió a Stigler sus simpatías por el mercado y el sistema capitalista, el cual decía "no será perfecto, pero es lo mejor entre las alternativas disponibles". Además de la influencia de Knight, durante esos años Stigler se acercó mucho a Jacob Viner, su profesor de teoría económica, y a Henry Simons, quien había escrito un plan de reforma de connotación liberal para rescatar la empresa privada de la Gran Depresión ${ }^{4}$.

En cuanto a Wallis, durante este período su interés académico era más bien en temas estadísticos, aunque en la práctica sentía una inclinación por incorporase al diseño y a la ejecución de políticas públicas. Aunque estos intereses le mantuvieron tibio con respecto a la filosofía de Chicago, Wallis nunca tuvo inclinaciones "socialistas" como las que manifiesta Friedman antes de su llegada a Chicago. Sus intereses estadísticos le llevaron a seguir los pasos de Friedman y a marcharse a Columbia en 1935, después de un breve ínterin en la Junta Nacional de Planificación en Washington.

Una vez concluido su programa de maestría, Friedman decidió poner fin a su segunda estadía en Chicago. Aunque nunca lo expresó explícitamente, su decisión de abandonar la universidad y probar suerte en otras latitudes tiene que ver más con sus finanzas y la situación familiar que con su futura carrera académica. Para un joven con ideas sociales, no del todo convencido de la religión de Chicago y con una situación económica precaria, el doctorado no era la mejor opción. Para entonces, como describe el propio Friedman, las becas graduadas que se daban en las grandes universidades cubrían costos de matrículas, pero no pagaban estipendios. Sus ingresos eran cercanos

\footnotetext{
${ }^{4}$ Ver Simons (1934), Un Programa Positivo de Laissez Faire.
} 
a cero, una situación insostenible precisamente cuando se acercaba la decisión de casarse con su novia de entonces, la también economista de Chicago Rose Director.

\section{El gran intervalo: diez años con trabajo y sin tesis doctoral}

\section{V.1. El análisis estadístico y los antecedentes de sus teorías de consumo e ingreso}

La abundante evidencia sobre la situación económica familiar y la impostergable necesidad de Friedman de generar recursos económicos explica no solo su partida de Chicago, sino también el largo período transcurrido antes del retorno definitivo a la academia que lo había formado. Diez años le tomó a Friedman desandar el camino. Abandonó la Universidad de Chicago en 1936, unos meses después del inicio de la revolución keynesiana con la publicación de la Teoría general del empleo, el interés y el dinero. Retornó de forma definitiva en 1946, luego de terminada la Segunda Guerra Mundial.

Sin duda, su experiencia laboral a lo largo de la década estuvo marcada por estos dos grandes eventos. Por un lado, la revolución keynesiana fue un elemento central para que se abrieran oportunidades de trabajo para jóvenes economistas en el gobierno de Estados Unidos. Por otro lado, la guerra hizo evidente la necesidad de contratar además de economistas, estadísticos y matemáticos capaces de utilizar los datos disponibles para eficientizar los recursos en una economía marcada por una conflagración mundial. Aunque Friedman aprovechó todo el espectro de oportunidades disponibles durante el tiempo que se mantuvo alejado de Chicago, su experiencia laboral no estuvo exenta de escollos.

Para comenzar, conseguir el primer trabajo fue un reto. Con los sucesos recientes en la vieja Europa, el sentimiento antisemita había ganado terreno en el mundo industrializado. Aunque en menor grado, Estados Unidos no había sido la excepción. Particularmente, en el 
sector privado de algunas ciudades, el origen semita de Friedman reducía sus probabilidades de conseguir un buen empleo. En contraste con las faltas de oportunidades privadas, el gobierno de Estados Unidos requería de jóvenes economistas dispuestos a trabajar en una de las múltiples agencias de planificación creadas en el marco del programa del presidente Roosevelt conocido como el Nuevo Trato (New Deal, en inglés).

La oportunidad llegaría rápidamente. Unos meses antes de que Friedman abandonara Chicago, su amigo Allen Wallis había logrado insertarse como economista en una de las agencias del Nuevo Trato, el Comité Nacional de Investigaciones (NRC, por sus siglas en inglés). Dentro de esta agencia, había sido asignado a un grupo dedicado fundamentalmente al estudio del consumo en los Estados Unidos, bajo el liderazgo de Hidelgarde Kneeland. En el siguiente verano, 1936, Wallis consiguió una pasantía a su antiguo compañero de universidad, una oportunidad que Friedman supo aprovechar, alcanzando unos meses después la condición de miembro oficial del Grupo Kneeland. Dos años de trabajo estadístico y análisis profundo de la base de datos de consumo más grande de los EE. UU. sentó las bases para el desarrollo de su famosa teoría de consumo, uno de sus grandes aportes a la teoría económica ${ }^{5}$.

En sus años de estadía con la NRC, Friedman fue cimentando su prestigio a tal punto que en 1937 fue escogido como representante del Grupo Kneeland en la conferencia anual del Bureau Nacional de Investigaciones Económicas (NBER). Una vez en Nueva York, Simon Kusnetz, futuro ganador del Premio Nobel de Economía, le reclutó para trabajar en el Bureau en temas de cuentas nacionales. Su primera asignación fue un estudio amplio sobre el ingreso de los profesionales liberales en los EE. UU., lo que significó una nueva oportunidad de

\footnotetext{
${ }^{5}$ Realmente el artículo seminal de Friedman sobre consumo se publicó dos décadas después, en 1957. Aunque en esos años se sentaron las bases para su escritura, en materia de publicación Friedman andaba por otro lado. Por ejemplo, en 1935 Friedman publicó un ensayo cuestionando la teoría de elasticidad de un economista reputado, Cecil Arthur Pigou. Por esas vueltas que da la vida, el primer intento de publicación de este estudio había sido enviado por Friedman a la revista científica Economic Journal, donde había sido rechazado por su editor, nada más y nada menos que John Maynard Keynes.
} 
acceder a otra base de datos de cardinal importancia, tal y como la había tenido con el consumo y el Grupo Kneeland. En esta ocasión, Friedman le sacaría aún más provecho a esa oportunidad.

El estudio profundo del ingreso de los profesionales liberales en los Estados Unidos le serviría para completar su tesis doctoral que sería defendida en la Universidad de Columbia, unos años después. A la vez, su exposición a las principales fuentes de información sobre el consumo y el ingreso de los estadounidenses le permitiría complementar su teoría de consumo con una teoría de ingreso permanente que colocaría la teoría keynesiana como un caso especial de consumo de corto plazo. Tanto en el caso del consumo como del ingreso, Friedman había construido sus teorías partiendo de una observación intensa de los datos que describían el comportamiento de los agentes económicos durante años. Este ejercicio se repetiría una y otra vez en su carrera, arrojando resultados positivos como el desarrollo del monetarismo a partir de su monumental obra Historia Monetaria de los Estados Unidos (1963).

Con el paso del tiempo, Friedman publicaría un artículo en el que explicaría su metodología para abordar los problemas económicos. Sin quererlo, este ensayo metodológico se convertiría en su obra más citada, y por qué no, en su trabajo más controversial (Friedman, 1953). Fundamentalmente, la metodología económica de Friedman valida una teoría, si y solo si, sus conclusiones no puedan ser refutadas por la evidencia empírica. En consecuencia, cuando la evidencia empírica corrobora las predicciones del modelo, poco importan los supuestos teóricos. En el mundo de la filosofía de las ciencias, Friedman sería un instrumentalista (Boland, 1979).

Durante la primera mitad de su periplo de diez años por el mercado de trabajo, Friedman pudo sembrar los cimientos de una carrera llamada a alcanzar la cúspide de la ciencia económica. No solo logró fortalecer sus habilidades estadísticas con el manejo de las grandes bases de datos de consumo e ingreso, sino que también pudo consolidar sus destrezas teóricas sentando las bases para más de un trabajo seminal en el campo de la economía. Pero, por encima de todos esos logros, su experiencia laboral con el consumo en la 
NRC y con el ingreso de los profesionales liberales en el NBER, le permitieron hacerse de una metodología económica que se llevaría consigo a Chicago cuando llegara la hora del viaje definitivo.

\section{V.2 Breve interludio académico y el retorno a Washington}

A pesar de casi un lustro dedicado a la investigación y al análisis estadístico, moviéndose entre una agencia gubernamental como la NRC y un tanque de pensamiento como la NBER, Friedman quería para sí una carrera académica. Esta es la única explicación para entender por qué en 1940, con seguridad económica y algún prestigio ganado, Friedman decidió quemar las naves y aceptar una posición en la facultad del pequeño Departamento de Economía de la Universidad de Wisconsin, en Madison. Cierto es que la posición era garantizada $\mathrm{y}$ ofrecía un gran salario, pero implicaba aventurarse a un mundo que le era desconocido.

Contrario a la seguridad que le había ofrecido Chicago y las ciudades donde había hecho vida a lo largo de la costa este de Estados Unidos, la Universidad de Wisconsin, localizada en el medio oeste norteamericano, no era particularmente amigable a sus orígenes judíos. De hecho, en la Escuela de Artes y Ciencias de Wisconsin, donde se ubicaba el Departamento de Economía, muchos docentes eran contrarios a la entrada de Estados Unidos en la Segunda Guerra Mundial, y otros, de orígenes germánicos, manifestaban abiertas simpatías con los alemanes. Cabe recordar que, al momento de la llegada de Friedman a Wisconsin, en 1949, Estados Unidos aún permanecía al margen del conflicto bélico global, aunque gradualmente se incrementaban las presiones para que entrara en acción respaldando a los aliados.

Más allá de las incomodidades que le generaron las pocas simpatías que despertaba su origen judío, Friedman se vio afectado por conflictos y luchas de egos que existían entre los miembros de la Facultad de Economía de la Universidad de Wisconsin. Particularmente, la oferta que se le hizo para enseñar estadísticas en Madison a través de Harold Grove, un profesor de finanzas públicas del Departamento de 
Economía de Wisconsin, tuvo sus detractores desde un principio. Tan pronto se enteraron de la contratación del nuevo docente, miembros de la facultad a cargo de las clases de estadísticas trataron de bloquear la entrada de Friedman a Wisconsin por miedo a perder sus posiciones ${ }^{6}$. Ante este contratiempo, a Friedman se le ofreció dictar cursos de matemáticas para economistas y de ciclos económicos.

El cambio de cátedra no le amilanó. Friedman montó sus cursos a pesar de no tener la oportunidad de enseñar estadística, como era su plan original. No obstante, el curso de matemáticas para economistas fue objeto de controversia debido a que el material preparado por Friedman para sus clases era de un nivel más alto que el que podían manejar la mayoría de los estudiantes que tomaron el curso con la formación que tenían. Ante este impasse, Friedman propuso al Departamento de Economía de Wisconsin exigir al menos una clase de cálculo como prerrequisito para tomar su curso, propuesta que no fue bien recibida por la facultad docente.

Con la inconformidad personal y familiar en el ambiente del medio oeste, los problemas académicos y, sobre todo, la alta carga antisemítica que sentía en la Universidad de Wisconsin, Friedman decidió renunciar a su empleo antes de cumplir el año. Así, en el otoño de 1941, optó por retornar a Washington, considerando la experiencia de Wisconsin como "el evento más traumático de su vida de formación durante sus años de joven economista" (Taylor, 2001).

\section{V.3 Una nueva experiencia laboral: la economía de la guerra}

Cuando Friedman tomó la decisión de irse a Wisconsin, en 1940, ya la Segunda Guerra Mundial había iniciado. Los nazis avanzaban con paso firme sobre Europa. Ya en el mes de junio habían ocupado gran parte del territorio francés y se preparaban para un inminente ataque a Inglaterra, uno de los pocos países del viejo continente que permanecía libre del dominio alemán. Mientras Europa sucumbía al dominio nazi, el presidente Roosevelt prometía, en un discurso de

\footnotetext{
${ }^{6}$ Según Hammond (2000), esos profesores eran Philip Fox y Erwin Gaumnitz.
} 
campaña en los Estados Unidos, que no enviaría tropas americanas a pelear en suelo extranjero.

A pesar del aislacionismo estadounidense, su economía no había salido ilesa del impacto de la conflagración mundial en casi todos los países europeos. Por esta razón, y mucho antes de que la primera potencia del mundo decidiera entrar en combate, el gobierno norteamericano incorporó a la administración pública un gran número de economistas con la tarea exclusiva de estudiar la economía de la guerra y hacer propuestas de política orientadas a suavizar el impacto del conflicto bélico en la economía de los Estados Unidos? ${ }^{7}$ Entre los economistas contratados por el tesoro americano estuvo Milton Friedman.

A su llegada a Washington, en el otoño de 1941, Friedman pasó a formar parte de un equipo en el Ministerio del Tesoro de los Estados Unidos cuya función principal era utilizar la política impositiva para evitar un proceso incontrolado de inflación durante la guerra. En ese entonces, la principal fuente de ingreso del gobierno norteamericano era el Impuesto sobre la Renta que cobraba trimestralmente el Tesoro, a través de su Servicio de Ingresos Internos (IRS, por sus siglas en inglés). Esta forma de cobrar el impuesto permitía el consumo de los ingresos individuales por un período de hasta tres meses antes de enfrentar las responsabilidades impositivas, lo que generaba presiones inflacionarias.

Con el objetivo de evitar el impacto de este gasto de consumo en la inflación, tal y como ocurrió en la Primera Guerra Mundial, Friedman y el grupo encargado de la política impositiva en las oficinas del Tesoro Americano propuso un mecanismo de retención de impuestos en la fuente, tan exitoso que aún hoy sigue vigente, no solo en Estados Unidos, sino en gran parte del mundo occidental. En adición a la propuesta de retención en la fuente, el grupo de Friedman en el tesoro elaboró el Acta Tributaria de 1942, una reforma que

\footnotetext{
${ }^{7}$ En el best seller internacional de la periodista económica Silvia Nasar se afirma que el número de economistas en Washington aumentó de un número cercano a 100, en 1930, a más de 5000, al comienzo de la Segunda Guerra Mundial. El número siguió aumentando con los empleos que generó la economía de la guerra. Ver Nasar (2011).
} 
proponía incrementos en el impuesto sobre la renta a las personas y a las empresas, como forma de financiar la economía de guerra.

A Friedman le tocó defender personalmente la reforma tributaria del Tesoro en el Congreso de los Estados Unidos. La experiencia fue interesante por representar dos hitos para el joven economista. En primer lugar, Friedman nunca se había presentado ante el Congreso para defender una propuesta de política económica. En segundo lugar, la propuesta que le tocó defender era keynesiana en toda su extensión, lo que indica qué tan lejos se encontraba Friedman de las ideas liberales en estos años.

Para confirmar esta aseveración, basta con revisar su testimonio al Congreso. Entre otras cosas, Friedman afirmó que "para reducir la inflación es necesario reducir el gasto de consumo" y dijo también que "el incremento de impuestos ayuda a lograr la reducción del consumo al disminuir el monto que los consumidores tienen disponibles para gastar". Citó como otros métodos para controlar la inflación "el control y el racionamiento de precios, el control del crédito a los consumidores y la reducción del gasto público"8. En todo el testimonio no se ve una alusión al dinero como determinante principal de la inflación. Friedman no era entonces monetarista. Como prueba de esta afirmación quedó la publicación de su análisis de cómo prevenir la inflación con impuestos, durante el período de la guerra (Shoup, C. S., Friedman, M., \& Mack, R. P., 1943).

\section{V.4 De la economía de la guerra a la estadística de la guerra}

En la segunda semana de diciembre de 1941, luego del ataque japonés a la fuerza naval de los Estados Unidos en Pearl Harbor, el presidente Roosevelt logró que el Congreso le aprobara de forma casi unánime una declaración de guerra a la nación oriental. Cuatro meses después, Alemania e Italia, aliados con Japón, declararon la guerra a Estados Unidos. La entrada de Estados Unidos al conflicto bélico mundial en la primera parte de 1942 cambió la visión de Washington

\footnotetext{
${ }^{8}$ Vease Friedman \& Friedman (1998, p. 111).
} 
sobre las tareas que debían realizar los economistas del gobierno. Del análisis de la economía de guerra y las propuestas de políticas, los economistas debían unirse a los equipos multidisciplinarios que se estaban preparando para explotar la información estadística disponible con miras a incrementar la eficiencia de las acciones de guerra de los aliados.

Esta información de tipo militar no era precisamente infalible, por lo que presentaba ciertas dificultades a los científicos naturales que cooperaban en la guerra, acostumbrados a utilizar datos de mejor calidad. No obstante, los economistas y los estadísticos, concentrados en el estudio de las ciencias sociales, estaban acostumbrados a trabajar con datos estadísticos imprecisos, lo que les daba una ventaja comparativa ante los científicos naturales como estadísticos de guerra (Wallis, 1980).

Conociendo estas ventajas, el vicepresidente de Ciencias Naturales del Centro Rockefeller, Warren Weaver, le ofreció una beca al estadístico San Wilks para realizar análisis de estadística de la guerra en la institución. Wilks, que había sido estudiante de Hotelling en Columbia, no tardó en recomendar a su antiguo profesor para encabezar un grupo que, financiado por el Centro Rockefeller, se dedicara al estudio de las estadísticas de la guerra con el objetivo de hacer más eficiente la acción en combate de los Estados Unidos. Hotelling aceptó y contactó a dos de sus antiguos alumnos, Allen Wallis y Jack Wolfowitz, para que se uniesen al proyecto. Así surge en el verano de 1942 el Grupo de Investigación Estadística (SRG, por sus siglas en inglés).

Las primeras reuniones del grupo fueron en casa de Hotelling en Mountain Lake, Nueva Jersey. Pero, poco tiempo después el SRG se trasladó a Nueva York, donde fue adquirido por la Universidad de Columbia. Ya instalados en un edificio cerca de Harlem, Allen Wallis invita a sus amigos de Chicago, Milton Friedman y George Stigler, a incorporarse al grupo. El SRG se mantuvo vigente entre 1943 y 1945, siempre al servicio de los estamentos armados de los Estados Unidos. Sus miembros se concentraron principalmente en el análisis estadístico de problemas relacionados con el diseño de armas, las tácticas militares 
y los experimentos metalúrgicos de distintos instrumentos de guerra9

Según Guglielmo (2008), el SRG se concentró en resolver problemas de guerra con el uso de tres tipos de técnicas: a) investigación operativa; b) inspección de muestras; y c) análisis secuencial. Varias de las tareas de Friedman en el SRG se circunscribieron al uso de la investigación operativa, es decir, de técnicas de optimización y otros métodos cuantitativos para estudiar las vulnerabilidades e ineficiencias de los aviones de guerra y proponer medidas que mejoren su efectividad en combate. En esa línea, calculó el número y tamaño óptimos de los componentes de un avión de guerra, así como de la cantidad y el calibre de sus armas. También estudió, con la intención de mejorar su efectividad, el fusible de proximidad de los aviones, un artefacto de radar que se coloca en la punta frontal del avión y que hace explosión cuando el enemigo se encuentra a cierta distancia ${ }^{10}$.

Dado que Friedman conocía la técnica de investigación operativa antes de unirse al SRG, su mayor aprendizaje en los años con el grupo fue su experiencia con la técnica estadística de análisis secuencial. Esta técnica que sería relevante en su futura carrera académica fue inventada por Abraham Wald, uno de los estadísticos de más renombre en el SRG. El análisis secuencial logró resolver uno de los grandes y más costosos problemas de la economía de la guerra.

Concretamente, para escoger un tipo de armamento de guerra (i.e. un proyectil) era necesario realizar una gran cantidad de pruebas para obtener una muestra que permitiera su aprobación. Con el análisis secuencial, los estadísticos de la SRG lograron realizar pruebas de hipótesis secuenciales, es decir, pruebas recurrentes en la medida que los datos estadísticos eran recabados. Esto, combinado con una regla preestablecida para detener el experimento cuando se tuviera suficiente información, permitió muestreos más cortos y mucho menos costosos.

No todo fue luz durante esta segunda estadía de Friedman en

\footnotetext{
${ }^{9}$ La historia de la fundación y las acciones del SRG está bien contada en Wallis (1980) y Olkin (1991).

${ }^{10}$ Friedman también estudió problemas de investigación operativa, relacionados con los esfuerzos para mejorar los métodos de búsqueda de submarinos enemigos durante la guerra.
} 
Columbia. Su experiencia como estadístico de guerra en el SRG también tuvo su cuota de ruidosos fracasos. Uno de ellos ocurrió cuando en 1944 el grupo le pidió a Friedman que estimara un modelo capaz de predecir el tiempo que podría soportar una aleación de metal en torno a la turbina de una aeronave de guerra al ser sometida a temperaturas extremas. Para cumplir con la tarea, Friedman diseñó un modelo de regresión múltiple donde el tiempo que podría soportar la aleación era función de variables como el nivel de la temperatura, la tensión a la que era sometida la aeronave y su composición metalúrgica.

Como describe Armstrong (2012), la estimación de la regresión que hoy se puede hacer en un segundo utilizando una computadora personal en ese entonces podía llevar meses de intensa labor. Por suerte para Friedman, el SRG tenía acceso a una computadora gigantesca asentada en el gimnasio aclimatado de la Universidad de Harvard que podía hacer los cálculos en menos de dos días. La ecuación de Friedman arrojó resultados significativos que llevaron al diseño de dos tipos de aleaciones que, según el modelo, serían capaces de sobrevivir cientos de horas a altas temperaturas. No obstante, cuando las aleaciones fueron testeadas en un laboratorio del Instituto Tecnológico de Massachusetts (MIT), ardieron antes de las tres horas.

Entre todas las luminarias que formaron parte del SRG, la figura de Friedman sobresalió como una de las más importantes. Friedman y Abraham Wald fueron los miembros del SRG más citados por sus colegas al preguntársele en un sondeo sobre las figuras más influyentes a lo interno del grupo en los años de la guerra (Wallis, 1980). Tal era su influencia que en una ocasión visitó la base de Los Álamos en Nuevo México, donde se hacían las pruebas de la bomba atómica en el marco del Proyecto Manhattan (Ebenstein, 2007, p. 45). Antes de esa visita y sin saber la naturaleza del trabajo realizado, Friedman había diseñado, junto a otros miembros del SRG, el procedimiento estadístico para asegurar un buen funcionamiento del detonador de la bomba.

En total, antes de su cierre definitivo en 1945, el SRG produjo más de 570 reportes, memos y cartas con los resultados de sus análisis. 
Friedman escribió una centena de ellos. Luego de un trabajo tan intenso, enmarcado dentro de las tensiones de la guerra, es probable que Friedman deseara más que nada volver a las aulas universitarias. En el fondo, ese era su hábitat natural. Por suerte, su amigo George Stigler había retornado antes del cierre del SRG al Departamento de Economía de la Universidad de Minnesota. Una vez terminada la guerra y a solicitud de Friedman, Stigler inició las gestiones para que su viejo amigo se le uniera en Mineápolis. El objetivo: enseñar estadísticas, más específicamente, análisis secuencial.

\section{EI retorno a Chicago: el viaje definitivo}

\section{VI.1 Minnesota: otra transición en el Medio Oeste}

Dada su terrible experiencia con el antisemitismo y los conflictos internos en los años de Wisconsin, resulta chocante que Friedman aceptara la oferta de la Universidad de Minnesota, situada a apenas 260 millas de distancia de su antiguo empleador. No obstante, su breve estadía en las ciudades gemelas fue radicalmente diferente a la experiencia vivida en Madison. En Minnesota fue feliz. Por un lado, su amistad con Stigler, con quien compartió oficinas, lo hizo sentirse como en casa. Por otro lado, ya más cerca de la ideología liberal, Friedman encontró varios colegas en el Departamento de Economía con los cuales tenía afinidades de ideas.

En cuanto al antisemitismo, aunque el ambiente en Minnesota no era necesariamente diferente al del Wisconsin de 1941, un hecho real había reducido las presiones contra los académicos de origen semita. Los aliados habían ganado la guerra. Por esta razón, en el campus universitario aquellos que pudiesen profesar simpatías por el "Eje del Mal" estaban replegados. En este entorno más moderado, Friedman enseñó estadísticas y economía y logró publicar, junto a su amigo Stigler, su famoso artículo contra el control de las rentas hipotecarias establecido en la guerra (Friedman \& Stigler, 1946).

Independientemente de estos logros, el período de estancia de Friedman en Minnesota fue corto. En apenas un año estaba haciendo 
sus maletas para marcharse a Chicago. Esta vez su partida fue el resultado de una oportunidad y no de una experiencia traumática como la que le llevó a abandonar Wisconsin un lustro antes. La Universidad de Chicago había tocado sus puertas con una oferta difícil de rechazar. Se le ofrecía la cátedra de Teoría de Precios que, durante décadas, había estado en manos de Jacob Viner, uno de los tres fundadores de la vieja escuela de Chicago.

No obstante, antes de abandonar Minnesota, Friedman completó dos tareas pendientes que le facilitaron su transición a la ciudad de los vientos. Primero, luego de años de controversia, la Universidad de Columbia aceptó como buena y válida su tesis doctoral basada en el trabajo que había hecho originalmente junto a Simon Kusnets en la NBER, sobre los ingresos de los profesionales liberales ${ }^{11}$. En segundo lugar, aprovechando la revisión que hacía George Stigler de su libro sobre teoría de precios, Friedman y su amigo mantuvieron discusiones intensas que le prepararon mejor para asumir la cátedra que habría de heredar con la partida de Jacob Viner a Princeton. Todo estaba listo para el viaje definitivo. Friedman volvía a Chicago.

VI.2 Chicago: comienza el viaje definitivo

Contrario a lo que generalmente se piensa, Friedman encontró a su regreso al Departamento de Economía de Chicago que la facultad se había alejado a pasos rápidos de las ideas liberales de sus mentores. La escuela en la que se había formado ya no existía. De sus antiguos líderes solo Frank Knight seguía en Chicago y su involucramiento con los asuntos departamentales era prácticamente nulo. Viner se había marchado a Princeton y Henry Simons había fallecido con apenas 46 años. Henry Schultz, quien había conseguido la beca a Friedman para estudiar en Columbia con Hotelling, se vio envuelto en un accidente automovilístico en California que resultó fatal.

\footnotetext{
${ }^{11}$ La controversia que surgió en torno a la tesis doctoral de Friedman está bien explicada en Ebenstein (2007, pp. 48-49). Todo comenzó por la exigencia de la Universidad de Columbia en aquellos años de que una tesis para ser aprobada debía primero ser publicada. El problema era que el trabajo de Friedman no había podido publicarse por una oposición levantada por la Asociación Médica Americana (AMA), debido a que en la tesis se argumentaba que las diferencias entre los salarios de los médicos y los de los dentistas se debía a que la AMA restringía la oferta de médicos en el mercado con sus políticas de entrada a la profesión.
} 
Tanto había cambiado el Departamento de Economía desde los tiempos en que Friedman obtuvo su maestría, que a su regreso a Chicago llenó la ausencia de quienes habían partido, con la presencia de aquellos que como él, regresaban al nicho académico luego de años de ausencia. Aaron Director, su cuñado, se incorporó a la Universidad de Chicago como profesor de la Escuela de Derecho, en 1946. Allen Wallis fue contratado como profesor de estadísticas, luego de una breve estadía en Stanford. Jimmy Savage, uno de sus más cercanos compañeros en el SRG y quien se convertiría en coautor de numerosas investigaciones, también se había incorporado al Departamento de Estadísticas.

No obstante, George Stigler, quien sería su copiloto en el viaje de relanzamiento de la ideología liberal en Chicago, tendría que esperar más de una década antes de poder retornar a la universidad. Stigler había partido de Minnesota a la Universidad de Brown luego de que el Departamento de Economía de Chicago rechazara su aplicación para incorporarse como docente a la facultad, supuestamente por considerársele un economista muy empírico ${ }^{12}$. Lo más irónico de este episodio es que la universidad le ofreció a Friedman la posición por la cual había rechazado a Stigler por sus inclinaciones "empíricas". A falta de su colega y amigo, Friedman tendría que echar solo sus primeras batallas por el dominio del Departamento de Economía. Su rival era un conglomerado de talentosos economistas, más influidos por las ideas keynesianas que por las ideas liberales.

En efecto, al retorno de Friedman a Chicago, en 1946, la visión filosófica más influyente en el Departamento de Economía era la promovida por un grupo compacto, la Comisión Cowles, específicamente un organismo de investigación económica creado en la década de 1930 del siglo pasado en el Estado de Colorado ${ }^{13}$.

\footnotetext{
${ }^{12}$ Oficialmente se dijo que su rechazo se originó en la administración de la universidad y que el Departamento de Economía lo quería como profesor. No obstante, es más probable, como dice su hijo, que el rechazo haya sido el resultado de la influencia de la Comisión Cowles en el Departamento de Economía y no de la administración universitaria. Véase Ebenstein (2007).

${ }^{13}$ Christ (1994) cuenta de forma detallada los orígenes de la Comisión y su influencia en el Chicago de los años cuarenta del siglo pasado.
} 
Alfred Cowles, un pudiente asesor de inversiones en los mercados financieros, se había propuesto utilizar su fortuna para entender lo que había ocurrido durante la Gran Depresión. Para cumplir este propósito, acogió una recomendación de un matemático que había conocido en Colorado Springs, Harold Davis, de que contactara al famoso economista Irving Fischer en la Universidad de Yale.

En ese entonces, Fischer era el economista americano vivo más importante. Con la fuerza de su prestigio, le propuso a Alfred Cowles la creación de una comisión de economistas notables y la publicación anual de una revista científica. Así nacieron la Comisión Cowles (CC) y la revista científica Econométrica, una de las publicaciones económicas más prestigiosas del mundo. Econométrica se convirtió en poco tiempo en la publicación insignia de la Sociedad Econométrica, una agrupación formada por Fischer y Ragnar Frisch en 1931 que tenía como objetivo el avance de la teoría económica y su relación con las estadísticas y las matemáticas. Los vínculos entre la CC y la Sociedad Econométrica en esta primera etapa eran más que obvios, ambos de alguna forma habían sido hijos de Irving Fischer.

Unos años después, en 1939, la Comisión Cowles aterrizó en Chicago para suplir el vacío estadístico que dejó la trágica muerte de Henry Schultz. Bajo el liderazgo de Jacob Marschack como director de investigaciones, la CC fue incorporando econometristas que, como Haavelmo, Koopsman, Arrow y Klein, obtendrían, uno por uno, el Premio Nobel de Economía. Con este capital humano, la comisión floreció y fue ganando prestigio. Su influencia en un Departamento de Economía que, por diversas circunstancias había perdido sus principales columnas, se tornó creciente y por momentos parecía que Chicago se rendiría al dominio keynesiano de la profesión. Es en este momento de preminencia de la CC que Friedman se une al Departamento de Economía.

Un detalle que a veces pasa desapercibido es que casi al mismo tiempo de su llegada a Chicago, Friedman había sido contactado por su antiguo profesor de Rutgers Arthur Burns, esta vez para formar parte de la NBER como investigador asociado. Friedman aceptó la oferta e inició lo que sería una fructífera relación con el Bureau, 
cuyo punto cumbre se alcanzaría con la publicación en 1963 de su Historia Monetaria de los Estados Unidos. Un par de años después, el conflicto que estaba a punto de iniciarse con la CC se agravaría cuando Koopsman, para entonces director de la comisión, atacara frontalmente a Wesley Clark Mitchell y al NBER.

Si alguna duda quedaba en Friedman y su círculo más íntimo sobre el rol que le tocaría jugar para arrebatar a Chicago a los economistas de la Comisión Cowles y convertirla en un refugio liberal, quedó disuelta antes de finalizar su primer año del retorno a la universidad. En esa fecha, Friedrich Hayek, el principal líder de la Escuela Austríaca, convocó a Friedman, junto a su cuñado Aaron Director, al encuentro que tendría lugar entre intelectuales liberales en la ciudad de Mount Pelerin, Suiza. Es ese encuentro el que oficializa la conversión liberal de Friedman que estaba de por sí muy avanzada. Fueron sus primeros contactos no solo con Hayek, sino también con Lionel Robbins y otros liberales europeos que, según sus palabras, le ayudaron a ampliar sus perspectivas de política económica (Doherty, 1995).

Friedman volvería de Mount Pellerin preparado para la batalla que se avecinaba con los economistas de la Comisión Cowles por el dominio del Departamento de Economía de Chicago. De esta colisión saldría triunfante la facción liberal que él encabezaba. El resto es historia conocida. Friedman se transformaría en el economista liberal más famoso del mundo y Chicago sería su centro de operaciones. Con sus colegas Stigler y Theodore Schultz llevaría a la cúspide a una escuela de economía que pariría, incluyéndolos, unos quince premios Nobel de economía.

El camino hacia el posicionamiento de sus ideas no siempre fue fácil. Su defensa acérrima del mercado le ganaría amigos y enemigos, éxitos e incomprensiones, pero sobre todo una alta influencia entre los hacedores de políticas de distintas partes del mundo. Por esta razón, hoy en día, pueden observarse huellas de sus principales tesis económicas en numerosos países. Esta es una realidad innegable, aunque muchos de los habitantes de estas naciones no comprendan hasta qué punto sus vidas están influenciadas por ideas que nacieron o se propagaron en el Chicago de Milton Friedman. 
Como explicara Lord Keynes en su Teoría general del empleo, el dinero y el interés (1936), "las ideas de los economistas y filósofos políticos, tanto cuando son correctas como erróneas, tienen más poder de lo que comúnmente se entiende. De hecho, el mundo está dominado por ellas. Los hombres prácticos, que se creen exentos de cualquier influencia intelectual, son usualmente esclavos de algún economista difunto". En el caso que nos ocupa, el economista se llama Milton Friedman y murió en 2006.

\section{Referencias}

Armstrong, J. S. (2012). Illusions in regression analysis. International Journal of Forecasting, 3(28), 689-694. DOI: https://doi. org/10.1016/j.jbusres.2015.03.029

Arrow, K. J. (1974). In Memoriam: Harold Hotelling, 1895-1973. American Economic Review, 64(6), 1102-1103. DOI: https:// doi.org/10.1111/j.1536-7150.1974.tb02444.x

Beyer, H. (1995). Selección de escritos políticos y económicos de Milton Friedman. Revista de Estudios Públicos, 60, 431-484.

Boland, L. A. (1979). A critique of Friedman's critics. Journal of Economic literature, 17(2), 503-522.

Christ, C. F. (1994). The Cowles Commission's contributions to econometrics at Chicago, 1939-1955. Journal of Economic Literature, 32(1), 30.

Doherty, B. (1995). Best of Both Worlds, Milton Friedman reminisces about his career as an economist and his lifetime "avocation" as a spokesman for freedom. Reason.

Ebenstein, L. (2007). Milton Friedman: a biography. New York, Estados Unidos: St. Martin's Press.

Frank H. Knight. (2002). Origins of the Chicago School of Economics. Economic Insights, 7, 1-4.

Frazer, W. J., \& Boland, L. A. (1983). An essay on the foundations of Friedman's methodology. The American Economic Review, 73(1), 129-144.

Friedman, M. (1935). Professor Pigou's method for measuring 
elasticities of demand from budgetary data. The Quarterly Journal of Economics, 50(1), 151-163. DOI: https://doi. org $/ 10.2307 / 1882346$

Friedman, M. (1953). The methodology of positive economics. Essays in positive economics, 3(3), 145-178. DOI: https://doi. org/10.1017/CBO9780511819025.010

Friedman, M., \& Schwartz, A. (1963). A Monetary History of the United States, 1867-1960. National Bureau of Economic Research, Inc. DOI: https://doi.org/10.2307/1917945

Friedman, M. (1976). Homer Jones: A personal reminiscence. Journal of Monetary Economics, 2(4), 433-436.

Friedman, M. (1977). Nobel lecture: inflation and unemployment. Journal of political economy, 85(3), 451-472. DOI: https://doi. org/10.1086/260579

Friedman, M.(1993). George Stigler:A personal reminiscence. Journal of Political Economy, 101(5), 768-773. DOI: https://doi. org $/ 10.1086 / 261898$

Friedman, M., \& Stigler, G. (1946). Roofs or ceilings. The Current Housing Problems, Popular Essays on Current Problems, 1(2).

Friedman, M., \& Friedman, R. D. (1998). Two lucky people: Memoirs. Chicago, Estados Unidos: University of Chicago Press.

Friedman, M., \& Robertson, D. H. (1973). A Milton Friedman-Sir Dennis Robertson Correspondence. Journal of Political Economy, 81(4), 1033-1039. DOI: https://doi. org/10.1086/260099

Garrison, R. W. (2014). Hayek and Friedman: Head to head. Elgar companion to Hayekian economics. Cheltenham and Northampton: Edward Elgar Publishing. CrossRef Google Scholar.

Guglielmo, M. (2008). The contribution of economists to military intelligence during World War II. The Journal of Economic History, 68(1), 109-150. DOI: https://doi.org/10.1017/ S0022050708000041

Hamilton, W. H. (1919). The institutional approach to economic theory. The American Economic Review, 9(1), 309-318. 
Hammond, J. D. (2000, January). Columbia roots of the Chicago school: the case of Milton Friedman. Unpublished paper. Wake Forrest University.

Keynes, J. M. (1936). The general theory of employment, interest and money (1936). Whitefish, Montana: Kessinger Publishing.

Krugman, P. (2007). Milton Friedman in China (Paul Krugman's' Who Was Milton Friedman?')-Reply. New York Review of Books, 54(6), 86-86.

Krugman, P. (2007). Who Was Milton Friedman? New York Review of Books, 54(2), 27.

Malthus, T. (2015). An Essay on the Principle of Population. Scotts Valley, CA: CreateSpace Publishing. (Trabajo original publicado en 1798).

Marx, K. (2011). Das Kapital (Samuel Moore, Trans.). Scotts Valley, CA: CreateSpace Publishing. (Trabajo original publicado en 1867).

McCloskey, D. (2003). Milton. Eastern Economic Journal, 29(1), 143-146.

Nasar, S. (2011). Grand pursuit: The story of economic genius. New York, Estados Unidos: Simon and Schuster.

Olkin, I. (1991). A conversation with W. Allen Wallis. Statistical Science, 6(2), 121-140. DOI: https://doi.org/10.1214/ ss/1177011818

Pérez, E. (2001). Milton Friedman. Comercio Exterior, 51(1), 15-30. Ricardo, D. (2004). The Principles of Political Economy and Taation. New York, Estados Unidos: Dover Publications. (Trabajo original publicado en 1817).

Samuelson, P. A. (1972). Jacob Viner, 1892-1970. Journal of Political Economy, 80(1), 5-11. DOI: https://doi.org/10.1086/259856

Schumpeter, J. (1954). History of economic analysis. New York, Estados Unidos: Oxford University Press.

Shoup, C. S., Friedman, M., \& Mack, R. P. (1943). Taxing to prevent inflation: techniques for estimating revenue requirements. New York: Columbia university press. DOI: https://doi. org/10.2307/137509 
Shleifer, A. (2009). The age of Milton Friedman. Journal of Economic Literature, 47(1), 123-35 DOI: https://doi.org/10.1257/ jel.47.1.123

Simons, H. C. (1934). A positive program for laissez faire: some proposals for a liberal economic policy. Chicago, Estados Unidos: University of Chicago Press.

Smith, A. (2004). The Wealth of Nations. Barnes \& Noble Library of Essential Reading. C. J. Bullock (ed.). New York, Estados Unidos: Barnes \& Noble Publishers. (Trabajo original publicado en 1776).

Taylor, J. B. (2001). An Interview with Milton Friedman. Macroeconomic Dynamics, 5(1), 101-131. DOI: https://doi.org/10.1017/S1365100501018053

Walker, M. (2006). Remembering Milton Friedman. FRASER INSTITUTE BOARD OF TRUSTEES Chairman, 3.

Wallis, W. A. (1980). The statistical research group, 1942-1945. Journal of the American Statistical Association, 75(370), 320330. DOI: https://doi.org/10.1080/01621459.1980.10477469

Weibel, C. (1995). A History of Mathematics at Rutgers. Extraído de http://sites.math.rutgers.edu/ weibel/history.html 